\title{
Prevention of peptic ulcers with esomeprazole in patients at risk of ulcer development treated with low-dose acetylsalicylic acid: a randomised, controlled trial (OBERON)
}

\author{
James M Scheiman, ${ }^{1}$ P J Devereaux, ${ }^{2}$ Johan Herlitz, ${ }^{3}$ Peter H Katelaris, ${ }^{4}$ \\ Angel Lanas, ${ }^{5}$ Sander Veldhuyzen van Zanten, ${ }^{6}$ Emma Nauclér, ${ }^{7}$ Lars-Erik Svedberg ${ }^{7}$
}

Division of Gastroenterology, University of Michigan Medical Center, Ann Arbor, Michigan, USA

${ }^{2}$ Department of Clinical Epidemiology and Biostatistics Joint Member, Department of Medicine (Cardiology),

McMaster University, Hamilton, Ontario, Canada

${ }^{3}$ Department of Molecular and Clinical Medicine, Sahlgrenska University Hospital, Gothenburg, Sweden

${ }^{4}$ Gastroenterology Department, Concord Hospital, University of Sydney, Sydney, Australia ${ }^{5}$ Department of Medicine, University Hospital, I+CS CIBERehd, Zaragoza, Spain ${ }^{6}$ Division of Gastroenterology, Department of Medicine, University of Alberta, Edmonton, Alberta, Canada

${ }^{7}$ AstraZeneca R\&D, Mölndal, Sweden

\section{Correspondence to}

James M Scheiman, Division of Gastroenterology, University of Michigan Medical Center, 3912 Taubman Center, SPC 5362. Ann Arbor, Ml 48109, USA; jscheima@med.umich.edu

Accepted 8 February 2011 Published Online First 17 March 2011

\begin{abstract}
Objective To determine whether once-daily esomeprazole $40 \mathrm{mg}$ or $20 \mathrm{mg}$ compared with placebo reduces the incidence of peptic ulcers over 26 weeks of treatment in patients taking low-dose acetylsalicylic acid (ASA) and who are at risk for ulcer development.

Design Multinational, randomised, blinded, parallelgroup, placebo-controlled trial.
\end{abstract}

Setting Cardiology, primary care and gastroenterology centres ( $n=240)$.

Patients Helicobacter pylori-negative patients taking daily low-dose ASA (75-325 mg), who fulfilled one or more of the following criteria: age $\geq 18$ years with history of uncomplicated peptic ulcer; age $\geq 60$ years with either stable coronary artery disease, upper gastrointestinal symptoms and five or more gastric/ duodenal erosions, or low-dose ASA treatment initiated within 1 month of randomisation; or age $\geq 65$ years. All patients were ulcer-free at study entry.

Interventions Once-daily, blinded treatment with esomeprazole $40 \mathrm{mg}$, $20 \mathrm{mg}$ or placebo for 26 weeks.

Main outcome measures The primary end point was the occurrence of endoscopy-confirmed peptic ulcer over 26 weeks.

Results A total of 2426 patients (52\% men; mean age 68 years) were randomised. After 26 weeks,

esomeprazole $40 \mathrm{mg}$ and $20 \mathrm{mg}$ significantly reduced the cumulative proportion of patients developing peptic ulcers; $1.5 \%$ of esomeprazole $40 \mathrm{mg}$ and $1.1 \%$ of esomeprazole $20 \mathrm{mg}$ recipients, compared with $7.4 \%$ of placebo recipients, developed peptic ulcers (both $p<0.0001$ vs placebo). Esomeprazole was generally well tolerated.

Conclusions Acid-suppressive treatment with once-daily esomeprazole $40 \mathrm{mg}$ or $20 \mathrm{mg}$ reduces the occurrence of peptic ulcers in patients at risk for ulcer development who are taking low-dose ASA

Clinical trial registration number ClinicalTrials.gov identifier: NCT00441727.

\section{INTRODUCTION}

For patients at increased cardiovascular risk, continuous low-dose acetylsalicylic acid (ASA, aspirin; 75-325 mg/day) reduces the risk of a range of cardiovascular events. ${ }^{1}{ }^{2}$ Low-dose ASA is a mainstay of protective treatment for secondary prevention of cardiovascular events, and is recommended for primary prevention in patients at high cardiovascular risk. ${ }^{3} 4$ In the most recent metaanalysis conducted by the Antithrombotic Trialists'
Collaboration, low-dose ASA was associated with a significantly lower risk of serious vascular events (eg, myocardial infarction, stroke or vascular death) in secondary cardiovascular prevention trials. ${ }^{1}$ In primary prevention trials, a significant reduction in serious vascular events was due mainly to a reduction in non-fatal myocardial infarction, whereas stroke and vascular mortality were not significantly reduced. The benefit in primary prevention trials was offset by a significant increase in the risk of major gastrointestinal and extracranial bleeding

Even at low doses, ASA is associated with gastrointestinal adverse effects, particularly in patients who are at increased gastrointestinal risk such as older patients and those with a previous history of ulcer disease. ${ }^{5}$ Low-dose ASA-associated adverse gastrointestinal effects range from troublesome upper gastrointestinal problems such as dyspeptic symptoms and heartburn ${ }^{6}$ to serious peptic ulcer complications, including gastrointestinal bleeding ${ }^{6}$ and perforated ulcers. ${ }^{9}$ Peptic ulcer complications have been reported to occur more than twice as frequently among low-dose ASA users than controls, ${ }^{10}{ }^{11}$ even among patients taking low-dose ASA for $>3$ months. ${ }^{12}$ The risk of gastrointestinal bleeding remains elevated with longer duration of ASA treatment ${ }^{13}$; indeed, the risk of gastrointestinal bleeding after 1 year of ASA use is more than double relative to non-users. ${ }^{11}$ Furthermore, the incidence of upper gastrointestinal bleeding has increased in parallel with the widespread use of low-dose ASA. ${ }^{14}$ Use of entericcoated or buffered formulations of low-dose ASA may not mitigate the risk of gastrointestinal complications. ${ }^{10}$ Gastrointestinal bleeding can occur without preceding symptoms, ${ }^{15}$ and may lead to discontinuation of low-dose ASA treatment. ${ }^{16}$ Adverse upper gastrointestinal symptoms with low-dose ASA use may lead to poor adherence to, or discontinuation of, low-dose ASA treatment ${ }^{17}$; this is worrying as discontinuation is associated with a markedly increased risk of serious adverse cardiovascular events that can occur within a few weeks. ${ }^{18}$ Therefore, continuing to treat patients at cardiovascular risk with long-term lowdose ASA is key in the management of cardiovascular disease. ${ }^{19}$ For these reasons, concomitant gastroprotection with a proton pump inhibitor (PPI) is recommended for cardiovascular patients who require continuous low-dose ASA and are at an increased gastrointestinal risk. ${ }^{20}$ online under the BMJ Journals unlocked scheme, see http:// heart.bmi.com/site/about/ unlocked.xhtml 
The OBERON trial explored primarily the efficacy of esomeprazole, at once-daily doses of $40 \mathrm{mg}$ and $20 \mathrm{mg}$, compared with placebo, in the prevention of peptic ulcers associated with continuous use of low-dose ASA in patients who were at an increased risk of developing peptic ulcers.

\section{PATIENTS AND METHODS}

OBERON was a randomised, blinded, parallel-group, placebocontrolled trial (ClinicalTrials.gov identifier: NCT00441727; AstraZeneca study code: D961FC00003), conducted at 240 cardiology, primary care and gastroenterology centres in 20 countries (Argentina, Australia, Bulgaria, Canada, Czech Republic, Finland, Germany, Indonesia, Mexico, Norway, Philippines, Poland, Portugal, Romania, Russia, Slovakia, South Africa, South Korea, Thailand and USA) in accordance with the Declaration of Helsinki and Good Clinical Practice guidelines. Patients, healthcare providers, data collectors and outcome adjudicators were blinded to treatment allocation. The study protocol was approved by the relevant local institutional review boards or independent ethics committees of each study centre, according to local regulations. All patients provided written, informed consent before enrolment in the trial. The first patient was randomised to the study on 22 February 2007 and the last patient completed the study on 28 August 2008.

\section{Patients}

Patients whose doctor prescribed or recommended daily use ( $\geq 5$ days/week) of low-dose ASA (75-325 mg/day) and who were Helicobacter pylori negative at screening were eligible to participate if they fulfilled one or more of the following inclusion criteria: aged $\geq 18$ years with documented history of uncomplicated peptic ulcer; aged $\geq 60$ years with one or more risk factor (stable coronary artery disease, or complaints of upper gastrointestinal symptoms that, as judged by the investigator, required an endoscopy resulting in a finding of five or more gastric and/or duodenal erosions at baseline endoscopy, or low-dose ASA-naïve (ie, treatment was initiated within 1 month of randomisation)); or aged $\geq 65$ years. Patients at very high cardiovascular and/or gastrointestinal risk were excluded from the study for ethical reasons. Cardiovascular exclusion criteria were unstable hypertension; recent (within 3 months) experience of acute coronary syndromes, percutaneous coronary intervention, coronary artery bypass grafting, clinically relevant valvular disease, serious cardiac failure (New York Heart Association class II-IV or ejection fraction $<40 \%$ ) and stroke. Gastrointestinal exclusion criteria were Los Angeles grade C-D erosive (reflux) oesophagitis at baseline; patient-reported severe oesophagitis within 1 year; peptic ulcer at baseline; history of peptic ulcer complications (eg, clinically significant bleeding and/or perforation) and previous gastric or duodenal surgery (patients who had undergone laparoscopic fundoplication were eligible). Other exclusion criteria were unstable diabetes mellitus: continuous treatment with a non-steroidal anti-inflammatory drug within 2 months of randomisation; ongoing anticoagulant treatment (antiplatelets such as clopidogrel were permitted); use of drugs that interact with esomeprazole (phenytoin, ketoconazole, itraconazole, voriconazole, cisapride, atazanavir, ritonavir); use of a PPI or prostaglandin analogue within 14 days of baseline endoscopy, or between baseline endoscopy and randomisation; daily use of histamine 2-receptor antagonists within 14 days of baseline endoscopy; and need for continuous treatment with prostaglandin analogues or sucralfate.

$H$ pylori tests were performed according to local routine practice and patients who were $H$ pylori positive had to have completed eradication treatment $\geq 4$ weeks before randomisation. Baseline $H$ pylori status was subsequently confirmed by a $\left[{ }^{13} \mathrm{C}\right]$ urea breath test and analysed by a central laboratory (Quintiles, Research Triangle Park, North Carolina, USA); confirmation test results were blinded to the investigator during the course of the study.

\section{Treatment and design}

After endoscopy and in addition to the low-dose ASA regimen prescribed by their doctor, eligible patients were randomised sequentially by numbered, opaque coded envelopes (in blocks of six) to 26 weeks' oral treatment with esomeprazole capsules at once-daily doses of $40 \mathrm{mg}$ or $20 \mathrm{mg}$, or placebo (in a ratio of 1:1:1). The randomisation codes were assigned from a computergenerated list held by the study sponsor (AstraZeneca R\&D, Mölndal, Sweden); code envelopes were used as an emergency unblinding tool. Esomeprazole and placebo capsules were supplied by the study sponsor and were identical in appearance and packaged in identical containers. Patients were instructed to take the study drug in the morning before breakfast with a glass of water. A rescue drug (antacids with acid-binding capacity of $<16 \mathrm{mmol} \mathrm{HCl} /$ tablet) was provided for use as needed ( $\leq 6$ tablets/day). Patients returned used study drug containers, rescue drug containers and all unused drugs to the study personnel at each study visit for assessment of drug adherence and rescue-drug use.

\section{Outcomes}

The primary end point was endoscopy-confirmed peptic (gastric or duodenal) ulcer during the 26-week treatment period. An ulcer was defined as a mucosal break measuring $\geq 3 \mathrm{~mm}$ over its largest diameter (size confirmed with endoscopy forceps) with a base (smooth or regular punched-out defect in the mucosa) and margin (discrete, sharply demarcated and usually raised in relation to the base) and the absence of any malignancy features. Endoscopy for investigation of peptic ulcers was performed at baseline, and at weeks 8 and 26 or upon withdrawal.

Secondary end points included the occurrence of a gastric ulcer and, separately, a duodenal ulcer, over 26 weeks of treatment, and safety and tolerability of treatments. Safety and tolerability during 26 weeks of treatment with esomeprazole or placebo were evaluated by assessments of adverse events and vital signs (including blood pressure and pulse rate), and by monitoring standard clinical laboratory tests and physical examinations. Spontaneously reported adverse events and those reported in response to a standardised question were recorded at all scheduled (weeks 8, 16 and 26) and unscheduled visits.

\section{Statistical analyses}

The primary analysis for the efficacy variables was based on the intention-to-treat (ITT) population, defined as all patients who were randomised. A supportive analysis for the primary end point was also conducted for the per-protocol population, which consisted of those patients in the ITT population with no major protocol violations, such as deviation from the inclusion/ exclusion criteria, or insufficient intake of the study drug.

The cumulative incidence of peptic, duodenal and gastric ulcer(s) was estimated from Kaplan-Meier life tables, and treatment groups were compared with placebo using the log-rank test stratified by ASA dose (75-100 mg and 101-325 mg). Patients who did not complete the 26 weeks for other reasons than the development of an ulcer were censored at the time of study discontinuation. Peptic, gastric and duodenal ulcers were tested in a hierarchical closed-test procedure, which was performed in 
parallel for the two esomeprazole doses with adjustment for multiplicity, using the Hochberg procedure, in each step. The effect of esomeprazole treatment on peptic ulcer incidence, stratified by ASA dose (75-100 $\mathrm{mg}$ and $101-325 \mathrm{mg}$ ), was analysed post hoc using Fisher's exact test. Analyses were conducted using SAS software (version 8.2; SAS Institute Inc).

\section{Sample-size calculation}

Based on data from a previous trial ${ }^{21}$ and the assumption that this study population had a higher gastrointestinal risk, we assumed an event rate for peptic ulcers in the placebo group and esomeprazole recipients of $8.0 \%$ and $3.2 \%$, respectively, corresponding to a relative risk reduction (RRR) of $60 \%$. We also assumed that $70 \%$ of the ulcers would be gastric. A sample size of 2400 patients ( 800 per treatment arm) was selected to provide $90 \%$ power for the primary end point (two-sided $\alpha=0.05$ ) at the $2.5 \%$ significance level, assuming a drop-out rate of $15 \%$.

\section{RESULTS}

\section{Patients}

A total of 2426 patients were randomised; all randomised patients were included in the ITT population as follows: esomeprazole $40 \mathrm{mg}(n=817)$, esomeprazole $20 \mathrm{mg}(n=804)$ or placebo $(n=805)$ (figure 1). Among the randomised patients, few (3.4\%) received concomitant clopidogrel treatment at any time during the study. Patient baseline characteristics were similar between treatment groups (table 1).

The per-protocol population included 1534 patients (esomeprazole $40 \mathrm{mg}$, 525 patients; esomeprazole $20 \mathrm{mg}$, 526 patients; placebo, 483 patients). The main reason for exclusion from the per-protocol population in all treatment groups was a positive $H$ pylori test at study conclusion or missing $H$ pylori status. The safety analysis included all patients who received one or more dose of investigational treatment and provided any follow-up data.

The majority (94\%) of patients were adherent (took $\geq 75 \%$ of doses) to esomeprazole. Patients used their own prescribed or

recommended low-dose ASA tablets on $\geq 5$ days/week, which were not counted.

\section{Incidence of ulcers}

Among the ITT population, the life table estimated cumulative peptic ulcer incidence over the 26 -week treatment period was $1.5 \%$ (95\% CI $0.6 \%$ to $2.4 \%$ ) in esomeprazole $40 \mathrm{mg}$ recipients, $1.1 \%$ (95\% CI $0.3 \%$ to $1.9 \%$ ) in esomeprazole $20 \mathrm{mg}$ recipients and $7.4 \%$ (95\% CI 5.5\% to $9.3 \%$ ) among placebo recipients (both $\mathrm{p}<0.0001$ vs placebo), resulting in an RRR versus placebo of $80 \%$ in esomeprazole $40 \mathrm{mg}$ recipients and $85 \%$ in esomeprazole $20 \mathrm{mg}$ recipients (figure 2), and an absolute risk reduction of $5.9 \%$ and $6.3 \%$, respectively. The result of the corresponding perprotocol analysis, from which $H$ pylori-positive patients were excluded, was consistent with the result of the ITT analysis. The observed incidence of peptic ulcer(s) in the ITT population was 11 esomeprazole $40 \mathrm{mg}$ recipients (1.3\%), 8 esomeprazole $20 \mathrm{mg}$ recipients $(1.0 \%)$ and 53 placebo recipients (6.6\%). The peptic ulcer incidence at 26 weeks in $H$ pylori-negative $(\mathrm{n}=1785)$ versus $H$ pylori-positive $(n=479)$ patients in the placebo arm was similar (6.8\% and 5.8\%, respectively). Esomeprazole $40 \mathrm{mg}$ and $20 \mathrm{mg}$ reduced the occurrence of peptic ulcer to $<2 \%$, relative to placebo, irrespective of $H$ pylori status.

Overall, gastric ulcers were more prevalent than duodenal ulcers in all treatment groups. Two placebo recipients with negative and missing $H$ pylori status, respectively, developed both duodenal and gastric ulcers. When ulcer incidence according to location was evaluated (data not shown), esomeprazole $40 \mathrm{mg}$ resulted in RRRs of $74 \%$ and $90 \%$ for gastric and duodenal ulcers, respectively, versus placebo, while the respective RRRs for esomeprazole $20 \mathrm{mg}$ were $83 \%$ and $90 \%$ (all $\mathrm{p}<0.0001$ vs placebo; ITT population).

In total, $79 \%$ of patients used daily low-dose ASA within the range of $75-100 \mathrm{mg}$. A post hoc analysis determined that esomeprazole $40 \mathrm{mg}$ and $20 \mathrm{mg}$ significantly reduced peptic ulcer incidence versus placebo in patients who used ASA within this range $(1.6 \%$ and $0.6 \%$ vs $6.1 \%$ of patients, respectively;

Figure 1 Patient flow through the study. All randomised patients are included in the intention-to-treat (ITT) analyses, including those patients who did not complete 26 weeks of treatment. Lack of response was defined as development of gastric and/or duodenal ulcer and/or upper gastrointestinal symptoms requiring active intervention; the study-specific discontinuation criterion was defined as low-dose acetylsalicylic acid (ASA) treatment permanently stopped; severe non-compliance was defined as all other forms of non-compliance except stopping low-dose ASA treatment; safety reasons were defined as those to protect study subjects from potential safety risks - for example, new data causing study termination or subpopulation of subjects to be discontinued. All discontinuations were judged by the study investigators. ${ }^{a}$ Two patients had positive Helicobacter pylori status and ulcer at baseline endoscopy; ${ }^{\mathrm{b}}$ six patients had Los Angeles(LA) grade $\mathrm{C}$ or $\mathrm{D}$ erosive (reflux) oesophagitis and ulcer at baseline endoscopy.

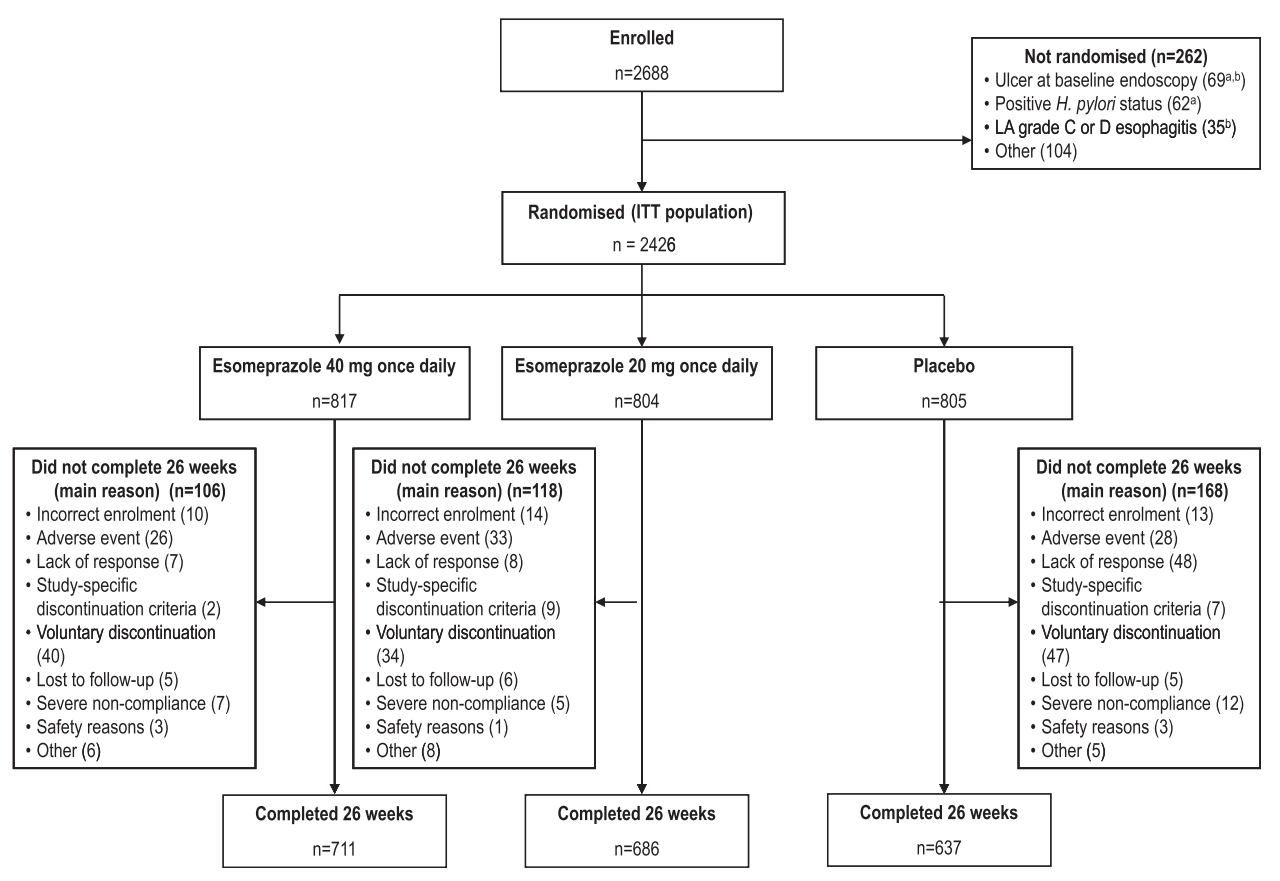


Table 1 Patient baseline characteristics (intention-to-treat population)

\begin{tabular}{|c|c|c|c|}
\hline \multirow[b]{2}{*}{ Characteristics } & \multicolumn{2}{|l|}{ Esomeprazole } & \multirow[b]{2}{*}{$\begin{array}{l}\text { Placebo } \\
(n=805)\end{array}$} \\
\hline & $\begin{array}{l}40 \mathrm{mg} \text { once } \\
\text { daily }(\mathrm{n}=817)\end{array}$ & $\begin{array}{l}20 \mathrm{mg} \text { once } \\
\text { daily }(\mathrm{n}=804)\end{array}$ & \\
\hline Men & $437(53.5)$ & $429(53.4)$ & $403(50.1)$ \\
\hline \multicolumn{4}{|l|}{ Age } \\
\hline Mean, years (range) & $67.7(21-87)$ & $67.7(24-89)$ & $67.4(24-94)$ \\
\hline$>70$ years & $299(36.6)$ & $286(35.6)$ & $282(35.0)$ \\
\hline \multicolumn{4}{|l|}{ Ethnicity } \\
\hline White & $669(81.9)$ & $651(81.0)$ & $661(82.1)$ \\
\hline Black/African-American & $30(3.7)$ & $31(3.9)$ & $23(2.9)$ \\
\hline Asian & $76(9.3)$ & $77(9.6)$ & $82(10.2)$ \\
\hline Other* & $42(5.1)$ & $45(5.6)$ & $39(4.8)$ \\
\hline Current smoker & $77(9.4)$ & $73(9.1)$ & $73(9.1)$ \\
\hline \multicolumn{4}{|l|}{ Helicobacter pylori status } \\
\hline H pylori negative & $606(74.2)$ & $605(75.2)$ & $574(71.3)$ \\
\hline H pylori positive & $159(19.5)$ & $149(18.5)$ & $171(21.2)$ \\
\hline $\begin{array}{l}\text { Missing/indeterminate } \\
\text { status }\end{array}$ & $52(6.4)$ & $50(6.2)$ & $60(7.4)$ \\
\hline History of gastric ulcer & $108(13.2)$ & $100(12.4)$ & $89(11.1)$ \\
\hline History of duodenal ulcer & $111(13.6)$ & $121(15.0)$ & $133(16.5)$ \\
\hline $\begin{array}{l}\text { History of erosive (reflux) } \\
\text { oesophagitis }\end{array}$ & $56(6.9)$ & $63(7.8)$ & $61(7.6)$ \\
\hline Past PCI & $112(13.7)$ & $103(12.8)$ & $102(12.7)$ \\
\hline Past CABG & $85(10.4)$ & $85(10.6)$ & $81(10.1)$ \\
\hline \multicolumn{4}{|l|}{ Low-dose ASA use } \\
\hline Primary prevention & $363(44.4)$ & $368(45.8)$ & $386(48.0)$ \\
\hline Secondary prevention & $435(53.2)$ & $421(52.4)$ & 401 (49.8) \\
\hline Mean dose, mg (range) & $112.1(75-325)$ & $109.2(50-325)$ & $107.7(75-325)$ \\
\hline $101-325 \mathrm{mg} / \mathrm{day}$ & $178(21.8)$ & $171(21.3)$ & $161(20.0)$ \\
\hline$>4$ weeks' duration & $713(87.3)$ & $684(85.1)$ & $700(87.0)$ \\
\hline
\end{tabular}

Variables are $\mathrm{n}(\%)$ unless otherwise stated.

*Including native Hawaiian/Pacific Islander, American Indian/Alaska native, Hispanic, Middle Eastern, Caribbean Island, Black/South African or mixed ethnicity.

ASA, acetylsalicylic acid; $C A B G$, coronary artery bypass grafting; $\mathrm{PCl}$, percutaneous coronary intervention.

$\mathrm{p}<0.0001)$. Similar findings were noted in patients who used ASA within the range of $101-325 \mathrm{mg}(0.6 \%$ and $2.3 \%$ vs $8.7 \%$ of patients, respectively; $\mathrm{p}<0.02$ ).

\section{Safety and tolerability}

Gastrointestinal complications

Few gastrointestinal complications were reported. By week 26, upper gastrointestinal complications were reported in two esomeprazole $20 \mathrm{mg}$ recipients (haematemesis $(n=1)$ and distal duodenal perforation thought to be associated with a known juxtapapillary diverticulum $(n=1))$, three placebo recipients (melaena $(n=2)$ and decreased haemoglobin level $(n=1)$ ), and no esomeprazole $40 \mathrm{mg}$ recipients.

\section{Other adverse events}

Overall, esomeprazole $40 \mathrm{mg}$ and $20 \mathrm{mg}$ were well tolerated, displaying similar tolerability profiles to placebo over 26 weeks of treatment. Adverse events were reported with a similar frequency in the three treatment groups (table 2). The most commonly reported adverse events were diarrhoea, headache and bronchitis. Overall, nine deaths occurred during the study (four esomeprazole $40 \mathrm{mg}$, four esomeprazole $20 \mathrm{mg}$ and one placebo recipient); however, none of these were considered by the study investigator at the centre to be causally related to the study drug. The causes of death (as reported by study investigators) were myocardial infarction ( $\mathrm{n}=2$ (one esomeprazole $40 \mathrm{mg}$ and one placebo recipient)), cerebrovascular accident (esomeprazole $20 \mathrm{mg}$ ), cardiac arrest (esomeprazole $20 \mathrm{mg}$ ), acute coronary syndrome (esomeprazole $20 \mathrm{mg}$ ), small bowel obstruction (esomeprazole $40 \mathrm{mg}$ ), acute renal failure (esomeprazole $40 \mathrm{mg}$ ), sudden death (esomeprazole $40 \mathrm{mg}$ ) and death from an unknown cause (esomeprazole $20 \mathrm{mg}$ ). The patient who died as a result of acute coronary syndrome began taking clopidogrel at the time of the cardiovascular event; none of the other patients who died were taking clopidogrel. Serious adverse events other than death were reported for $5.3 \%$ of esomeprazole $40 \mathrm{mg}, 4.9 \%$ of esomeprazole $20 \mathrm{mg}$ and $4.4 \%$ of placebo recipients; again, none of these were considered to be causally related to the study drug. There were no cardiovascular events in patients receiving clopidogrel, and no further events in patients who experienced a cardiovascular event and began taking clopidogrel during the trial $(n=5)$. The incidence of adverse events categorised as cardiac disorders was low, and there were no clinically meaningful differences in the incidence of these events among the treatment groups $(2.5 \%, 2.4 \%$ and $2.1 \%$ for esomeprazole $40 \mathrm{mg}$, $20 \mathrm{mg}$ and placebo, respectively).

\section{DISCUSSION}

In the OBERON trial, once-daily esomeprazole $40 \mathrm{mg}$ and $20 \mathrm{mg}$ significantly reduced the incidence of endoscopy-confirmed peptic ulcer, compared with placebo, during 26 weeks of treatment. Esomeprazole had a similar effect among patients taking ASA between the ranges of $75-100 \mathrm{mg}$ and $101-325 \mathrm{mg}$.
Figure 2 Cumulative percentage of patients with peptic ulcer(s) by week 26 (intention-to-treat population, Kaplan-Meier curve).

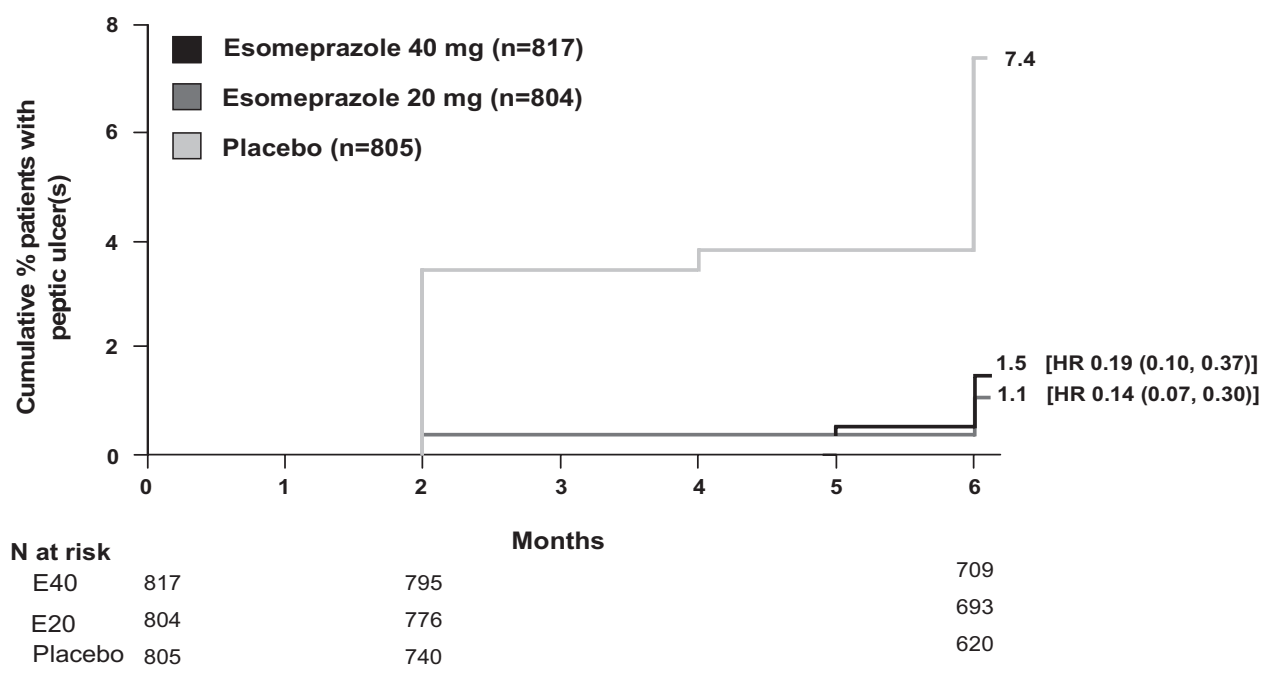


Table 2 Number (\%) of patients with adverse events (safety population)

\begin{tabular}{|c|c|c|c|}
\hline \multirow[b]{2}{*}{ Patients with adverse events } & \multicolumn{2}{|l|}{ Esomeprazole } & \multirow[b]{2}{*}{$\begin{array}{l}\text { Placebo } \\
(\mathrm{n}=801)\end{array}$} \\
\hline & $\begin{array}{l}40 \mathrm{mg} \text { once } \\
\text { daily }(\mathrm{n}=814)\end{array}$ & $\begin{array}{l}20 \mathrm{mg} \text { once } \\
\text { daily }(\mathrm{n}=799)\end{array}$ & \\
\hline Any adverse events, $\mathrm{n}(\%)$ & $295(36.2)$ & $297(37.2)$ & $298(37.2)$ \\
\hline Fatal serious adverse events, $\mathrm{n}(\%)^{*}$ & $4(0.5)$ & $4(0.5)$ & $1(0.1)$ \\
\hline $\begin{array}{l}\text { Non-fatal serious adverse } \\
\text { events, } \mathrm{n}(\%)^{*}\end{array}$ & $43(5.3)$ & $39(4.9)$ & $35(4.4)$ \\
\hline $\begin{array}{l}\text { Adverse events leading to } \\
\text { treatment withdrawal, } \mathrm{n}(\%)\end{array}$ & $30(3.7)$ & $37(4.6)$ & $42(5.2)$ \\
\hline $\begin{array}{l}\text { Treatment-related adverse } \\
\text { events, } \mathrm{n}(\%)\end{array}$ & $34(4.2)$ & $39(4.9)$ & $31(3.9)$ \\
\hline \multicolumn{4}{|l|}{ Most common adverse events, $\mathrm{n}(\%)$} \\
\hline Diarrhoea & $21(2.6)$ & $27(3.4)$ & $18(2.2)$ \\
\hline Headache & $15(1.8)$ & $15(1.9)$ & $15(1.9)$ \\
\hline Bronchitis & $11(1.4)$ & $17(2.1)$ & $14(1.7)$ \\
\hline Upper abdominal pain & $13(1.6)$ & $7(0.9)$ & $15(1.9)$ \\
\hline Influenza & $11(1.4)$ & $9(1.1)$ & $13(1.6)$ \\
\hline Back pain & $8(1.0)$ & $9(1.1)$ & $14(1.7)$ \\
\hline Nasopharyngitis & $7(0.9)$ & $13(1.6)$ & $10(1.2)$ \\
\hline Upper respiratory tract infection & $7(0.9)$ & $8(1.0)$ & $15(1.9)$ \\
\hline Dizziness & $7(0.9)$ & $9(1.1)$ & $12(1.5)$ \\
\hline Nausea & $15(1.8)$ & $6(0.8)$ & $6(0.7)$ \\
\hline Constipation & $9(1.1)$ & $10(1.3)$ & $6(0.7)$ \\
\hline Hypertension & $10(1.2)$ & $8(1.0)$ & $7(0.9)$ \\
\hline Urinary tract infection & $6(0.7)$ & $10(1.3)$ & $8(1.0)$ \\
\hline Dyspepsia & $7(0.9)$ & $3(0.4)$ & $12(1.5)$ \\
\hline
\end{tabular}

*No serious adverse event was considered by the investigators to be causally related to the study drug.

Esomeprazole in combination with low-dose ASA was well tolerated and there were no safety concerns. The data from the OBERON trial support and extend the findings of the earlier ASTERIX trial, which demonstrated the efficacy of esomeprazole $20 \mathrm{mg}$ in reducing the incidence of peptic ulcers associated with continuous use of low-dose ASA. ${ }^{21}$

The results of the OBERON trial are potentially clinically relevant in several ways. Although there is a recognised difference between endoscopic ulcers and bleeding complications, endoscopic ulcers have been used as clinical markers of gastrointestinal end points, based on the rationale that prevention of ulcer occurrence would reduce the incidence of ulcer bleeds. ${ }^{22}$ Indeed, there is evidence that concomitant PPIs decrease hospitalisations due to serious gastrointestinal bleeding in patients taking ASA or non-steroidal anti-inflammatory drugs. ${ }^{23}$ More recently, the Clopidogrel and Optimisation of Gastrointestinal Events Trial (COGENT) showed that prophylactic PPI treatment significantly reduced the rate of upper gastrointestinal bleeding among patients receiving dual antiplatelet treatment, without a significant increase in the risk of cardiovascular events. $^{24}$

Peptic ulcers with the potential for gastrointestinal bleeding may influence underlying cardiovascular disease-for example, peptic ulcers can cause anaemia, ${ }^{25}$ an independent risk factor for mortality and hospitalisation in patients with myocardial infarction. ${ }^{26}$ Also, gastrointestinal bleeding is independently associated with mortality and ischaemic complications in patients with acute coronary syndrome. ${ }^{27}$

The results of the OBERON trial extend the findings of ASTERIX, providing data that are relevant to clinical practice. By refining the inclusion criteria for recruiting patients with higher gastrointestinal risk than those patients who took part in ASTERIX, OBERON provides data on low-dose ASA users who are most likely to benefit from concomitant PPIs. Of note, the risk factors for gastrointestinal bleeding are also important risk factors for coronary disease. ${ }^{1}$ Patients with multiple erosions at baseline were included in the study as an increased rate of gastric/duodenal erosions was previously associated with lowdose ASA treatment. ${ }^{28}$ Patients who were naïve to low-dose ASA treatment were also included, as epidemiological data indicate that patients treated with ASA develop peptic ulcers at a higher rate during the initial treatment phase (within 1 month) than after the initial phase. ${ }^{11}$ However, our documented incidence of peptic ulcer development is still likely to be conservative; higher ulcer rates have been found in low-dose ASA users elsewhere. Patients with the highest gastrointestinal risk (baseline peptic ulcers, reflux oesophagitis and a history of peptic ulcer complications) and cardiovascular risk were excluded from this study for ethical reasons.

The benefit of concomitant PPI treatment in a subgroup of low-dose ASA recipients at very high gastrointestinal risk, using ulcer complications as end points, has previously been demonstrated. $^{29}$ Of particular relevance to patients at increased cardiovascular risk, concomitant low-dose ASA and esomeprazole treatment significantly lowered the risk of recurrent ulcer bleeding compared with clopidogrel monotherapy. ${ }^{30}$ Given these data, it is not surprising that the use of PPIs for gastroprotection among patients with gastrointestinal risk factors who require low-dose ASA or clopidogrel for cardiovascular risk management is supported by expert opinion in the joint statement by the American College of Cardiology Foundation, American College of Gastroenterology and the American Heart Association. ${ }^{20}$ More than $20 \%$ of the ITT population were $H$ pylori positive (as determined by a $\left[{ }^{13} \mathrm{C}\right]$ urea breath test), indicating a high level of false-negative serology screening tests at the study centres. A similar rate of false-negative tests was also noted during the ASTERIX trial. In order to preserve the gastrointestinal risk profile of the patients, the result of the confirmatory test was not provided to the investigator during the study unless requested. Since $H$ pylori testing is not always performed in routine clinical practice before starting antiplatelet treatment, the data support the efficacy of the treatment in the target population.

Reduction of ulcer development in low-dose ASA users was recently demonstrated with use of an $\mathrm{H} 2$-receptor antagonist (H2-RA), famotidine, $20 \mathrm{mg}$ twice daily for 3 months. ${ }^{31}$ Many methodological differences between this and our study make comparisons difficult; of particular note, over $20 \%$ of patients in the famotidine study did not have final endoscopic confirmation of ulcer status. Furthermore, $42 \%$ of placebo recipients who developed ulcers had $H$ pylori infection, versus no instances of infection in the active treatment group, raising the possibility that many of the ulcers prevented were attributable to $H$ pylori infection and not to low-dose ASA. Further, in a longer-term (48 weeks) trial, famotidine $40 \mathrm{mg} /$ day was shown to be inferior to a PPI (pantoprazole) in the prevention of dyspeptic or bleeding ulcers/erosions in patients with ASA-related peptic ulcers/erosions. ${ }^{32}$ Concerns about the long-term use of H2-RAs for the reduction of low-dose ASA-related ulcers include tachyphylaxis, as well as lack of convincing observational data that bleeding ulcers can be reduced, leading to recommendations favouring PPI over H2-RA treatment. ${ }^{20}$ While the efficacy of H2-RA treatment in reduction of gastric bleeding has been observed, the effect was less than has been shown with PPI treatment. ${ }^{33}$ The efficacy of esomeprazole $40 \mathrm{mg}$ and $20 \mathrm{mg}$ was shown in an OBERON analysis for both the $H$ pylori-negative population and patients proved to be $H$ pylori positive after randomisation. The same observation was made in the ASTERIX study, which used esomeprazole $20 \mathrm{mg}$ daily. $^{21}$ 
In conclusion, the results of this trial demonstrate that esomeprazole $40 \mathrm{mg}$ and $20 \mathrm{mg}$ once daily reduces the risk of endoscopically proved peptic ulcer in patients who take low-dose ASA, and who are at risk for ulcer development. In the population studied, the esomeprazole $40 \mathrm{mg}$ and $20 \mathrm{mg}$ regimens appear to be equally effective.

Acknowledgements The authors thank Anna Mett and Jackie Campbell from inScience Communications, a Wolters Kluwer business, who wrote the first draft of this paper based on direction from the authors. Medical writing services from inScience Communications were funded by AstraZeneca, the manufacturer of esomeprazole. Statistical analyses were conducted and validated by EN (AstraZeneca R\&D, Mölndal, Sweden) and PJD (McMaster University, Hamilton, Ontario, Canada),observed via web-based link reproduction of the statistical analyses.

Funding This study was supported by AstraZeneca, the manufacturer of esomeprazole.

Competing interests JMS—consultant: AstraZeneca, Novartis, Pfizer, Bayer, Takeda, Pozen, NiCox. Speaker's honoraria: AstraZeneca. PJD—AstraZeneca previously supplied the study drug for a large, international, investigator-initiated, Canadian Institutes of Health Research funded randomised, controlled trial (ie, the POISE-1 Trial). JH-no conflicts of interest declared. PHK—speaker for AstraZeneca, Nycomed and Janssen-Cilag. No stocks or shares or direct financial links. AL-adviser to AstraZeneca, Pfizer, Nicox, Bayer. Speaker: AstraZeneca, Pfizer.

SVvZ - grant/research support and/or honoraria: Abbott, AstraZeneca, Janssen-Ortho, Nycomed and Takeda. EN, L-ES-employees: AstraZeneca.

Ethics approval This study was conducted with the approval of the relevant local institutional review boards or independent ethics committees of each study centre, according to local regulations.

Provenance and peer review Not commissioned; externally peer reviewed.

\section{REFERENCES}

1. Baigent C, Blackwell L, Collins $R$, et al. Aspirin in the primary and secondary prevention of vascular disease: collaborative meta-analysis of individual participant data from randomised trials. Lancet 2009;373:1849-60.

2. Patrono C, Bachmann F, Baigent C, et al. Expert consensus document on the use of antiplatelet agents. The task force on the use of antiplatelet agents in patients with atherosclerotic cardiovascular disease of the European society of cardiology. Eur Heart J 2004;25:166-81.

3. Pearson TA, Blair SN, Daniels SR, et al. AHA Guidelines for Primary Prevention of Cardiovascular Disease and Stroke: 2002 Update: Consensus Panel Guide to Comprehensive Risk Reduction for Adult Patients Without Coronary or Other Atherosclerotic Vascular Diseases. American Heart Association Science Advisory and Coordinating Committee. Circulation 2002;106:388-91.

4. US Preventive Services Task Force. Aspirin for the prevention of cardiovascular disease: U.S. Preventive Services Task Force recommendation statement. Ann Intern Med 2009;150:396-404.

5. Hernandez-Diaz S, Garcia Rodriguez LA. Cardioprotective aspirin users and their excess risk of upper gastrointestinal complications. BMC Med 2006;4:22

6. Hayden M, Pignone M, Phillips C, et al. Aspirin for the primary prevention of cardiovascular events: a summary of the evidence for the U.S. Preventive Services Task Force. Ann Intern Med 2002;136:161-72.

7. Yeomans ND, Lanas Al, Talley NJ, et al. Prevalence and incidence of gastroduodenal ulcers during treatment with vascular protective doses of aspirin. Aliment Pharmacol Ther 2005;22:795-801.

8. Serrano $\mathbf{P}$, Lanas A, Arroyo MT, et al. Risk of upper gastrointestinal bleeding in patients taking low-dose aspirin for the prevention of cardiovascular diseases. Aliment Pharmacol Ther 2002;16:1945-53.

9. Taha AS, Angerson WJ, Prasad R, et al. Clinical trial: the incidence and early mortality after peptic ulcer perforation, and the use of low-dose aspirin and nonsteroidal anti-inflammatory drugs. Aliment Pharmacol Ther 2008;28:878-85.

10. Sorensen HT, Mellemkjaer L, Blot WJ, et al. Risk of upper gastrointestinal bleeding associated with use of low-dose aspirin. Am J Gastroenterol 2000;95:2218-24.

11. Lanas A, Garcia-Rodriguez LA, Arroyo MT, et al. Risk of upper gastrointestinal ulcer bleeding associated with selective cyclo-oxygenase-2 inhibitors, traditional non-aspirin non-steroidal anti-inflammatory drugs, aspirin and combinations. Gut 2006;55:1731-8.

12. Lanas A, Bajador E, Serrano P, et al. Nitrovasodilators, low-dose aspirin, other nonsteroidal antiinflammatory drugs, and the risk of upper gastrointestinal bleeding N Engl J Med 2000;343:834-9.

13. Lanas A, Perez-Aisa MA, Feu F, et al. A nationwide study of mortality associated with hospital admission due to severe gastrointestinal events and those associated with nonsteroidal antiinflammatory drug use. Am J Gastroenterol 2005:100:1685-93.

14. Taha AS, Angerson WJ, Prasad R, et al. Upper gastrointestinal bleeding and the changing use of COX-2 non-steroidal anti-inflammatory drugs and low-dose aspirin. Aliment Pharmacol Ther 2007;26:1171-8.

15. Armstrong CP, Blower AL. Non-steroidal anti-inflammatory drugs and life threatening complications of peptic ulceration. Gut 1987:28:527-32

16. CAPRIE Steering Committee. A randomised, blinded, trial of clopidogrel versus aspirin in patients at risk of ischaemic events (CAPRIE). Lancet 1996;348:1329-39.

17. Pratt S, Thompson VJ, Elkin EP, et al. The impact of upper gastrointestinal symptoms on nonadherence to, and discontinuation of, low-dose acetylsalicylic acid in patients with cardiovascular risk. American Journal of Cardiovascular Drugs 2010;10:281-8.

18. Biondi-Zoccai GG, Lotrionte $\mathrm{M}$, Agostoni $\mathrm{P}$, et al. A systematic review and meta-analysis on the hazards of discontinuing or not adhering to aspirin among 50,279 patients at risk for coronary artery disease. Eur Heart J 2006;27:2667-74

19. Herlitz J, Toth PP, Naesdal J. Low-dose aspirin therapy for cardiovascular prevention: quantification and consequences of poor compliance or discontinuation. Am J Cardiovasc Drugs 2010;10:125-41.

20. Abraham NS, Hlatky MA, Antman EM, et al. ACCF/ACG/AHA 2010 expert consensus document on the concomitant use of proton pump inhibitors and thienopyridines: a focused update of the ACCF/ACG/AHA 2008 expert consensus document on reducing the gastrointestinal risks of antiplatelet therapy and NSAID use. Am J Gastroenterol 2010;105:2533-49.

21. Yeomans $\mathbf{N}$, Lanas A, Labenz J, et al. Efficacy of esomeprazole (20 mg once daily) for reducing the risk of gastroduodenal ulcers associated with continuous use of low-dose aspirin. Am J Gastroenterol 2008;103:2465-73.

22. Moore A, Bjarnason I, Cryer B, et al. Evidence for endoscopic ulcers as meaningful surrogate endpoint for clinically significant upper gastrointestinal harm. Clin Gastroenterol Hepatol 2009;7:1156-63.

23. Rahme E, Nedjar H, Bizzi A, et al. Hospitalization for gastrointestinal adverse events attributable to the use of low-dose aspirin among patients 50 years or older also using non-steroidal anti-inflammatory drugs: a retrospective cohort study. Aliment Pharmacol Ther 2007;26:1387-98.

24. Bhatt DL, Cryer BL, Contant CF, et al. Clopidogrel with or without omeprazole in coronary artery disease. N Engl J Med 2010;363:1909-17.

25. Annibale B, Capurso G, Chistolini A, et al. Gastrointestinal causes of refractory iron deficiency anemia in patients without gastrointestinal symptoms. Am J Med 2001;111:439-45.

26. Anker SD, Voors A, Okonko D, et al. Prevalence, incidence, and prognostic value of anaemia in patients after an acute myocardial infarction: data from the OPTIMAAL trial. Eur Heart J 2009;30:1331-9.

27. Nikolsky E, Stone GW, Kirtane AJ, et al. Gastrointestinal bleeding in patients with acute coronary syndromes: incidence, predictors, and clinical implications: analysis from the ACUITY (Acute Catheterization and Urgent Intervention Triage Strategy) trial. J Am Coll Cardiol 2009;54:1293-302.

28. Laine L, Maller ES, Yu C, et al. Ulcer formation with low-dose enteric-coated aspirin and the effect of COX-2 selective inhibition: a double-blind trial. Gastroenterology 2004:127:395-402.

29. Lai KC, Lam SK, Chu KM, et al. Lansoprazole for the prevention of recurrences of ulcer complications from long-term low-dose aspirin use. $N$ Engl J Med 2002;346:2033-8.

30. Chan FK, Ching JY, Hung LC, et al. Clopidogrel versus aspirin and esomeprazole to prevent recurrent ulcer bleeding. N Engl J Med 2005;352:238-44.

31. Taha AS, McCloskey C, Prasad R, et al. Famotidine for the prevention of peptic ulcers and oesophagitis in patients taking low-dose aspirin (FAMOUS): a phase III, randomised, double-blind, placebo-controlled trial. Lancet 2009;374:119-25.

32. $\mathbf{N g} \mathbf{F H}$, Wong SY, Lam KF, et al. Famotidine is inferior to pantoprazole in preventing recurrence of aspirin-related peptic ulcers or erosions. Gastroenterology 2010;138:82-8.

33. Lanas A, Garcia-Rodriguez LA, Arroyo MT, et al. Effect of antisecretory drugs and nitrates on the risk of ulcer bleeding associated with nonsteroidal anti-inflammatory drugs, antiplatelet agents, and anticoagulants. Am J Gastroenterol 2007:102:507-15 